\title{
Structural dynamics and inhibitor searching for Wnt-4 protein using comparative computational studies
}

This article was published in the following Dove Press journal:

Drug Design, Development and Therapy

29 April 2015

Number of times this article has been viewed

\section{Mirza A Hammad \\ Syed Sikander Azam}

National Center for Bioinformatics, Quaid-i-Azam University, Islamabad, Pakistan

Correspondence: Syed Sikander Azam National Center for Bioinformatics, Quaid-i-Azam University, Islamabad 45320, Pakistan

Tel +92 5l 90644130

Email ssazam@qau.edu.pk
Abstract: Wnt-4 (wingless mouse mammary tumor virus integration site-4) protein is involved in many crucial embryonic pathways regulating essential processes. Aberrant Wnt-4 activity causes various anomalies leading to gastric, colon, or breast cancer. Wnt-4 is a conserved protein in structure and sequence. All Wnt proteins contain an unusual fold comprising of a thumb (or N-terminal domain) and index finger (or C-terminal domain) bifurcated by a palm domain. The aim of this study was to identify the best inhibitors of Wnt-4 that not only interact with Wnt-4 protein but also with the covalently bound acyl group to inhibit aberrant Wnt-4 activity. A systematic computational approach was used to analyze inhibition of Wnt-4. Palmitoleic acid was docked into Wnt-4 protein, followed by ligand-based virtual screening of nearly 209,847 compounds; conformer generation of 271 compounds resulted from extensive virtual screening and comparative docking of 10,531 conformers of 271 unique compounds through GOLD (Genetic Optimization for Ligand Docking), AutoDock-Vina, and FRED (Fast Rigid Exhaustive Docking) was subsequently performed. Linux scripts was used to handle the libraries of compounds. The best compounds were selected on the basis of having maximum interactions to protein with bound palmitoleic acid. These represented lead inhibitors in further experiments. Palmitoleic acid is important for efficient Wnt activity, but aberrant Wnt-4 expression can be inhibited by designing inhibitors interacting with both protein and palmitoleic acid.

Keywords: thumb-index fold, comparative study, natural products, inhibitor searching, cancer, molecular docking, virtual screening

\section{Introduction}

Wnt-4, or wingless mouse mammary tumor virus integration site-4, is a hydrophobic, glycosylated, and acylated signaling protein that regulates many embryonic processes in a paracrine manner. ${ }^{1,2}$ When expressed normally, Wnt-4 regulates the polar cell polarity pathway, ${ }^{3}$ the $\beta$-catenin pathway, ${ }^{4}$ and the $\mathrm{Ca}^{2+}$ pathway, ${ }^{5}$ which collectively affect regulation of neuronal axons, ${ }^{6}$ kidney formation, ${ }^{7-9}$ development of the mammary glands, ${ }^{10}$ eye development, ${ }^{11}$ and male to female sex reversal. ${ }^{12,13}$ Aberrant expression of Wnt-4 may disturb these pathways, causing many abnormalities, including colon, breast, and gastric cancers. ${ }^{14} \mathrm{Wnt}-4$ protein has a hydrophobic nature due to the presence of a high number of hydrophobic amino acids. Palmitoylation not only amplifies the hydrophobicity of Wnt-4, but is also functionally important. Non-palmitoylated Wnt-4 is not able to exit the endoplasmic reticulum. ${ }^{15}$ Our lack of understanding of the intricate nature of the Wnt family, makes it difficult to crystallize its members. Since the initial discovery of the Int-1 protein, ${ }^{16}$ only two crystal structures has been resolved. ${ }^{17,18}$

Wnt proteins are believed to be animal proteins because they are only present in invertebrates and vertebrates. ${ }^{19}$ Wnt proteins are highly conserved in terms of their 
sequence and folding mechanism. Another distinctive feature of the Wnt proteins is the presence of cysteine residues, which exist mostly as cysteine-cysteine disulfide bonds that are also conserved in number and position. ${ }^{10}$ The crystal structure of Xwnt-8 reveals a highly unfamiliar fold with a distinct geometry, which has not been seen in previous protein structure studies. The protein is observed to mimic a thumb and index finger bulging out of a palm, where the thumb represents an N-terminal domain (NTD) and the index finger represents a C-terminal domain (CTD). Both the NTD and CTD contain fewer numbers of amino acids with a hydrophilic nature, whereas the palm contains an ample number of amino acids, mostly of a hydrophobic nature. Palmitoleic acid is also present at the thumb domain, and is important in the Wnt-frizzled interaction. According to National Center for Biotechnology Information (http://www. ncbi.nlm.nih.gov/protein/NP_110388.2) and UniProt (http:// www.uniprot.org/uniprot/P56705) records, the Wnt-4 protein contains a total of 351 amino acids, with the first 22 amino acids operating as a signal peptide and the remaining 329
(23-351) amino acids operating as a mature peptide. Most of the amino acid residues are hydrophobic (Figure 1). The mature Wnt- 4 peptide contains 24 cysteine residues that may be involved in cysteine-cysteine disulfide bridges.

\section{Wnt-4 pathway}

The Wnt-4 signaling pathway is of key importance in the development and progression of cancer in all animals. In the course of this pathway, Wnts target nearly 125 proteins, and this number is set to grow as research on these genes continues (http://www.stanford.edu/group/nusselab/cgibin/wnt/target_genes). Wnt-4 invokes many pathways in which there is a major contribution by frizzled receptors. Wnt-4 is expressed in the embryonic and developmental stages, and continues to be expressed in adulthood. The most important pathways are canonical and non-canonical, where the canonical pathway leads to gene transcription and the non-canonical pathway maintains the cytoskeleton. ${ }^{8,20}$ Non-canonical Wnt-4 signaling is also important for the development of the eye in Xenopus laevis. ${ }^{11}$ Wnt-4 acts as

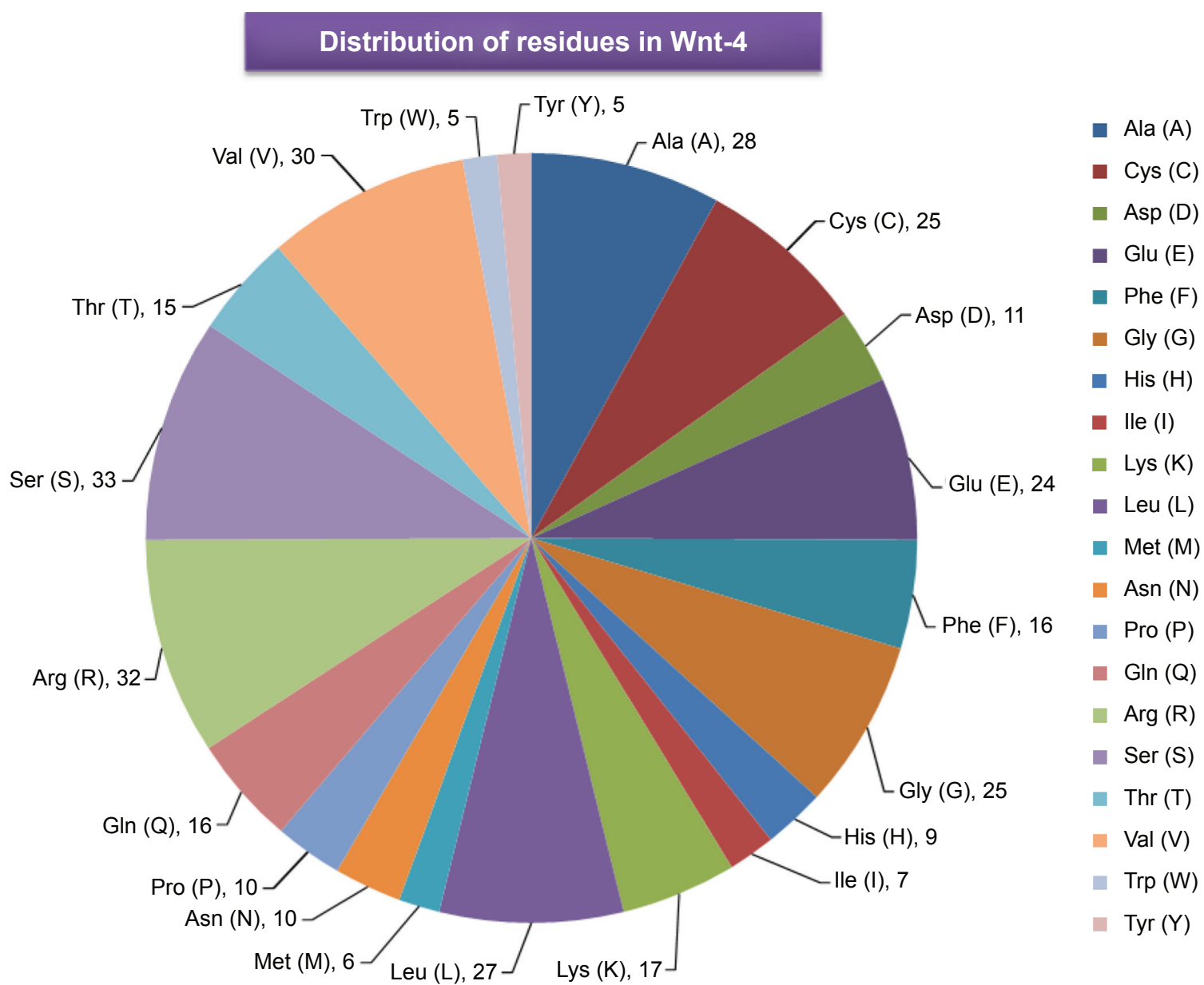

Figure I Distribution of residues along mature peptide of Wnt-4 protein. Wnt proteins are usually very hydrophobic in nature with few hydrophilic characteristics. Wnt-4 also has more hydrophobic residues that make it a conserved protein. 
a signal for nearly 14 proteins that further regulate cellular activity. ${ }^{21}$ After its translation, Wnt-4 is directed toward the endoplasmic reticulum for post translational modification. The most important post translational modifications include glycosylation and acylation, which impart increased activity to Wnt-4. PORCN is responsible for transferring the acyl group and for palmitoylating Wnt-4. This palmitoylation adds extra hydrophobicity, which enables Wnt-4 to survive outside the cell. A multipass transmembrane, Wntless, is responsible for transport and secretion of Wnt-4.

Despite its clinical importance in diagnosis and as a crucial signaling protein, no direct inhibitors of Wnt-4 have as yet been reported. It is quite difficult to directly inhibit Wnt-4 activity because of its high level of structural complexity. ${ }^{22}$ Most investigations have been aimed at Wnt-inhibiting mechanisms, ie, indirect inhibition of Wnt-4 activity by targeting proteins involved in its pathway. ${ }^{23}$ Wnt-inhibiting mechanisms involve signaling feedback to inhibit the activity of Wnt, but may also induce other anomalies, since the targeted protein might be significant in many biological processes. There is only one example of direct in vivo inhibition of Wnt, ie, liposomally packed antagonists to mouse Wnt-3a. ${ }^{24}$ These antagonists could act as a template and provide a basis for designing direct Wnt inhibitors.

In this study, we searched for direct inhibitors of Wnt-4 protein using computational techniques, including virtual screening (VS) and comparative docking. For this purpose, we used antagonists as a template for searching out potent small-molecule inhibitors of Wnt-4 protein.

Computational studies have had some success in increasing our understanding of complex biological processes, including signaling pathways, structural dynamics, and fold kinetics. ${ }^{25,26}$ With advances in technology, new algorithms are being developed for VS and docking, which have increased the search space for discovery of bioactive compounds and docking of large libraries of compounds in a comparative fashion. ${ }^{27}$ VS identifies compounds by screening large databases and employing knowledge about protein structure (structure-based VS) or known bioactive compounds (ligand-based VS). Docking enables prediction of the structure of a protein-inhibitor complex under equilibrium conditions. ${ }^{28}$ Posing and ranking are the major outputs of VS and docking techniques. The pose score measures the ability of a ligand to fit into the active site of a protein, while the rank score is more complex but attempts to estimate binding energies. ${ }^{29-31}$

In a previous study, we investigated the Wnt-4 thumbindex fold by applying molecular dynamics simulation techniques. ${ }^{32}$ Simulation of molecular dynamics has provided a method for studying the time-dependent dynamic behavior of biomolecules in an aqueous environment. ${ }^{33}$ Simulation of molecular dynamics integrates the Newtonian model into all the molecules of a system and utilizes a model based on the Ergodic hypothesis to generate images for each time step. ${ }^{34,35}$ From our analysis, we argued that cysteine residues are important for stabilization of the thumb-index fold of the Wnt-4 protein. Whereas importance and physicochemical properties of cysteine residues have also been reported in a previous study. ${ }^{32} \mathrm{We}$ also tend to find out the answer to a question of Willert and Nusse ${ }^{3}$ regarding the uncomplexed structure of Wnt proteins.

The thumb-index fold and acylation are important functional paradigms related to Wnt-4 activity. Acylation functionally enhances Wnt-4 activity and enables Wnt-4 to interact with frizzled receptors. Palmitoleic acid and palmitic acid are two major candidates for acylation of Wnt proteins. Wnt-4 was docked with both compounds to ascertain which compound was the most probable acylating factor. The acylated Wnt-4 was used in further experiment as a receptor. A consistent approach was applied to search for Wnt-4 inhibitors, including VS of 209,847 natural product compounds from the ZINC database, ${ }^{36}$ followed by comparative docking techniques. Large-scale searching for inhibitors by VS with subsequent comparative docking would assist effective discovery of the interrelationships between potent inhibitors, and the structural, kinetic, and thermodynamic properties of Wnt-4 protein.

\section{Materials and methods}

Due to limited availability of resources and the subtle nature and increasing number of proteins, it is not always possible to analyze proteins at a structural level. Further, atomic level details of energy changes between folding and unfolding of a protein are currently not possible to discover using in vivo and/or in vitro techniques. ${ }^{37-39}$ Therefore, for such proteins, the application of computational techniques like homology modeling and molecular dynamics simulation are the only methods by which structural information can be obtained. ${ }^{40,41}$ A systematic approach was defined and utilized that involved acquiring the structure of Wnt-4 protein followed by VS and docking to identify inhibitors (Figure 2). For the current study, the Wnt-4 structure was retrieved from the Protein Model Database (ID PM0078954), as previously identified and submitted by Azam and Hammad. ${ }^{32}$ The structure of Wnt-4 was used in further experimentation for molecular docking of small molecules. The small molecules were retrieved from the ZINC database. VS was performed to identify the best compounds for docking. 


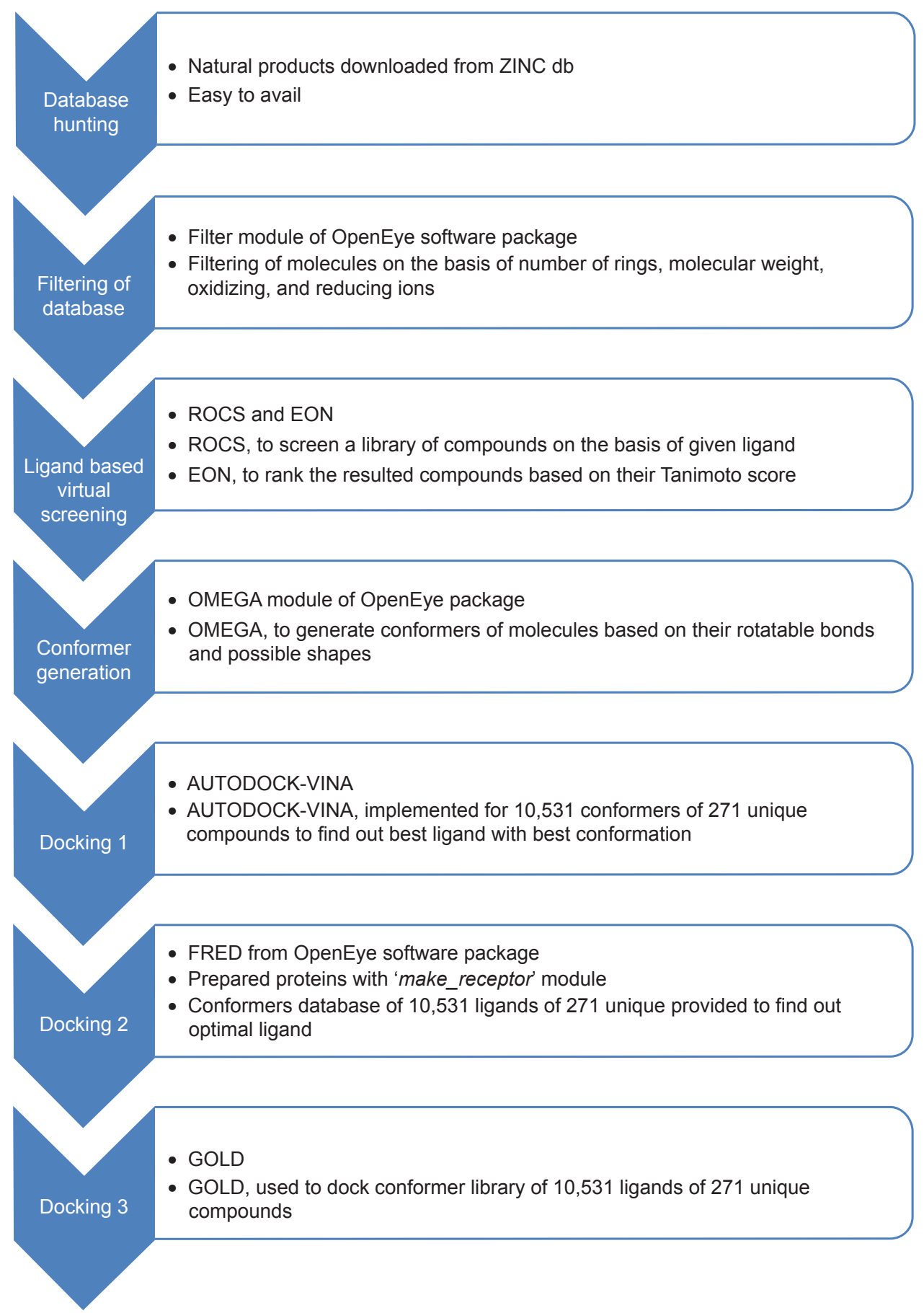

Figure 2 Flow diagram of virtual screening and docking of Natural Products. Major virtual screening was done using the OpenEye software package. Virtual screening was led by docking through AutoDock-Vina, FRED, and GOLD.

Abbreviations: FRED, Fast Rigid Exhaustive Docking; GOLD, Genetic Optimization for Ligand Docking.

\section{Virtual screening}

Using the correct parameters, VS shortens the inhibitor search time by screening large databases. VS was performed to search for the most effective inhibitors that might stabilize aberrant expression of Wnt-4. The most part of VS was performed using the OpenEye scientific software package. ${ }^{42}$ The FILTER, ROCS, EON, and OMEGA tools from this software package were used. Based on fast molecular filtering and selection, FILTER heuristically accelerates compound property calculation and removes undesirable compounds prior to entering the VS step. ${ }^{43}$ The FILTER tool was used to filter the whole database of natural products. The filters were applied such that molecules would follow Lipinski's rule of five and remove any metal-containing compounds. 
This filtering identifies the molecules having the most potent drug-like activity. FILTER shortened the compound library to 58,533 compounds, and ligand-based VS was performed for Wnt-4 protein. Currently, no inhibitor is reported for Wnt-4 protein, so an inhibitor for a closely related protein was considered.

The antagonist of mouse Wnt-3a was used as a template ligand for ROCS to search the library of compounds resulting from the FILTER tool. The ROCS tool is used in ligand-based VS for shape similarity and lead hopping. ${ }^{44,45}$ ROCS was used to screen compounds on the basis of the given template and returned a library of 8,109 compounds. At the first attempt, ROCS was used on eleven separate filtered natural product libraries. These libraries were then merged and ROCS was used a second time, resulting in 5,000 compounds. The ROCS library was subjected to EON, which compares electrostatic potential maps of preordinate compounds and specifies Tanimoto measures for the comparison. ${ }^{46}$ It ranked the compounds on the basis of their Tanimoto and shape scores. EON does not perform VS, but does accelerate it. It is used with ROCS to sort and rank compounds according to their electrostatic similarity. ${ }^{47,48}$ Compounds having an EON score $>0.85$ were considered to best match the template or antagonist, and separated 271 compounds. ${ }^{46,49}$ To obtain the best results from the docking experiment, conformers of compounds were generated. The OMEGA tool is widely recognized for its high speed and its ability to generate reliable multi-conformer ensembles encompassing bioactive conformations of the library of compounds. ${ }^{50}$ The 271 compounds including the template were subjected to OMEGA to generate an archive of conformers. ${ }^{50} \mathrm{~A}$ maximum limit of 200 conformers was applied for each compound (ringed compounds produce many more conformers due to the presence of a maximum rotatable bond). OMEGA generated a library of 10,531 conformers with distinct geometry.

\section{Docking}

In view of the importance of palmitoylation for Wnt-4 activity, the comparative docking step included protein with palmitoleic acid bound at Ser190 (Supplementary materials 1). In acylation of a protein, the acylated compound is covalently attached to the protein. Therefore, the Protein Data Bank (pdb) file of Wnt-4 was manually edited to covalently attach palmitoleic acid through the CONECT keyword. In the current study, the term "receptor" refers to a protein with palmitoleic acid attached. Comparative docking studies provide better results and deliver a consensus for a particular study. Comparative docking was performed using three different tools, ie, FRED, AD-VINA, and GOLD.

\section{FRED}

FRED (Fast Rigid Exhaustive Docking) is a fast and effective docking tool with significantly more reliability and lower variance. ${ }^{51}$ FRED scrutinizes all potential poses by performing an organized, extensive, and non-stochastic analysis of compounds. Based on shape complementarity, compounds are filtered, and based on pharmacophoric features, poses are selected and optimized. The Chemgauss4 scoring function is implemented to score and rank compounds. ${ }^{52}$ In a threedimensional world, molecules may change their conformation during interaction, whereas FRED is better for rigid docking. To overcome this issue, compounds were made rigid and conformers of compounds generated by OMEGA were used. Only the palmitoleic acid and Ser190 of the Wnt-4 receptor were made flexible. After preparation of the Wnt-4 receptor, a library of compounds was also prepared for utilization in FRED. Preparation of a receptor and a library of compounds improves the speed and efficiency of FRED. Compounds were docked utilizing the Chemgauss4 scoring function. A log file was generated, which ranked compounds on the basis of the highest Chemgauss 4 score.

\section{AutoDock}

AutoDock is designed to predict binding affinities of small molecules, including substrates and drug candidates, for a known three-dimensional protein structure. Currently, two AutoDock generations are available, ie, AutoDock 4 (AD-4) and AutoDock Vina (AD-VINA). While in comparison with $\mathrm{AD}-4, \mathrm{AD}-\mathrm{VINA}$ has a higher average accuracy and speed, ${ }^{53}$ AD-VINA, like AD-4, uses pdbqt of protein and ligands. The pdbqts are pdb files with added columns of Gasteiger charges. ${ }^{54,55}$ AD-VINA uses the Monte Carlo algorithm to dock compounds and ranks compounds based on their binding affinity values. ${ }^{27}$ Linux scripts were prepared to convert pdbs of the Wnt-4 receptor and library of compounds into pdbqt format. As with FRED, AD-VINA is also better for rigid docking, and performs docking efficiently if molecules are kept rigid. Therefore, no compound was made flexible, except for the palmitoleic acid and Ser190 of the Wnt-4 receptor, and previously generated conformers of OMEGA were used for docking purposes. With default parameters, AD-VINA docked a library of 10,531 compounds into the binding site of the Wnt-4 receptor. Log files were generated that ranked all compounds with the highest binding affinities. 


\section{GOLD}

For the purpose of acquiring the best compounds using the comparative docking approach, the library of compounds utilized for AD-VINA and FRED was also used for docking through GOLD (Genetic Optimization for Ligand Docking). GOLD has proven success in VS, lead optimization, and identifying the correct binding mode of bioactive compounds. ${ }^{56}$ It implements a genetic algorithm that incorporates the concept of chromosomes for building the best models of compounds involved in the docking process. ${ }^{57}$ Although GOLD has an intrinsic ability to generate a library of conformers, OMEGA conformers were used here and all small molecules were made rigid. Palmitoleic acid and Ser190 were made flexible to obtain a consensus in all the docking experiments. The compounds were docked with the Wnt-4 receptor binding site using the Goldscore scoring function and redocked using the Chemscore scoring function. This generated log files that were used to sort and rank compounds on the basis of Goldscore and Chemscore scoring functions.

OMEGA had identified 271 unique compounds including a template and generated 10,531 conformers. Scripting was drilled to isolate the best docked conformer of each compound through all docking tools. Compounds were compared to select the best inhibitor of the Wnt-4 protein. The comparison was based primarily on the highest Chemgauss 4 scores, the binding affinity, and the Goldscore, and secondly; on the ability and number of interactions made with the receptor and inhibitor. Chimera and LigPlot2 were used to identify the interactions of the compounds with the Wnt-4 receptor.

\section{Results}

The structure of Wnt-4 (ID PM0078954) was retrieved from the Protein Model Database. ${ }^{58}$ Analysis of the structure of Wnt-4 showed that it has two distinct domains: one lying at the NTD and other at the CTD, with a region of hydrophobic residues in between. The two domains, NTD and CTD, were comparable with the thumb and index finger joined by a hydrophobic region resembling the palm. The protein was observed to make an unusual thumb-index fold, previously revealed in the crystal structure of XWnt- $8 .{ }^{17}$ Analysis of the Wnt-4 structure also revealed the presence of 24 cysteine residues, mostly lying near the thumb (NTD) and index (CTD) domains (Figure 3). The cysteine residues could exist in disulfide bridges and stabilize the unusual thumb-index fold. Analysis of Wnt-4 with an XWnt-8 crystal structure also revealed that the point of acylation for Wnt-4 is Ser190. Acylation is important for Wnt activity because it enables Wnts to interact with frizzled receptors.
It also adds extra hydrophobicity to the Wnt molecule. ${ }^{10}$ Non-acylated Wnt may partially or completely lose its activity, and in some cases be restricted to the endoplasmic reticulum. ${ }^{59,60}$ If acylation is inhibited or the acyl compound is restricted by another inhibitor compound, Wnt proteins may not correctly interact with the frizzled receptor. There is no evidence of a direct inhibitor of Wnt-4 protein in the literature or the known databases. In the current study, an attempt was made to inhibit aberrant Wnt-4 expression by designing a small-molecule inhibitor for Wnt-4 including an acyl group. For inhibitor searching, ligand-based VS was performed utilizing an antagonist of mouse Wnt-3a as a template. For acylation of Wnts, the most potent candidates are palmitic acid (16-carbon) and palmitoleic acid (18-carbon). Both compounds were comparatively docked to the Ser190 position through docking programs including FRED, AD-VINA, and GOLD (Supplementary materials 1). The comparison-based resulting compound, palmitoleic acid, was covalently attached to Wnt-4 for further experiments.

\section{Virtual screening}

The Natural Products database was used for VS. Natural products are always a source of good drugs for scientific research and provide a better foundation for designing new drug candidates. ${ }^{61,62}$ Natural products also provide a number of phytochemicals that are currently being used as lead compounds in many drug design studies. ${ }^{63}$ The Natural Products database consists of nearly 209,847 small molecules from eleven different sources. Prior to VS and docking, the Natural Products database was filtered for any metal-containing compounds and heavy compounds with multiple rings. The FILTER tool from the OpenEye package was used for this purpose. Default parameters were used to scrutinize 58,533 compounds, following Lipinski's rule of five. These compounds were subjected to the ROCS tool for ligand-based screening of compounds against the template antagonist. Ligand-based searching through ROCS resulted in nearly 5,000 compounds based on the shape Tanimoto score. Selection of compounds is an important step, and requires better representation of compounds with correct parameters. The EON tool from the OpenEye package was used for this purpose, and not only sorted compounds based on their shape Tanimoto score, but also considered their charge properties. This combination score is known as the ET Combo (coulombic electrostatic Tanimoto indices) and has a maximum value of $1 .{ }^{64,65}$ All compounds with an EON ET combo value $\geq 0.850$ were selected. ${ }^{49}$ ET Combo indexing of EON screened 271 unique compounds, including an 


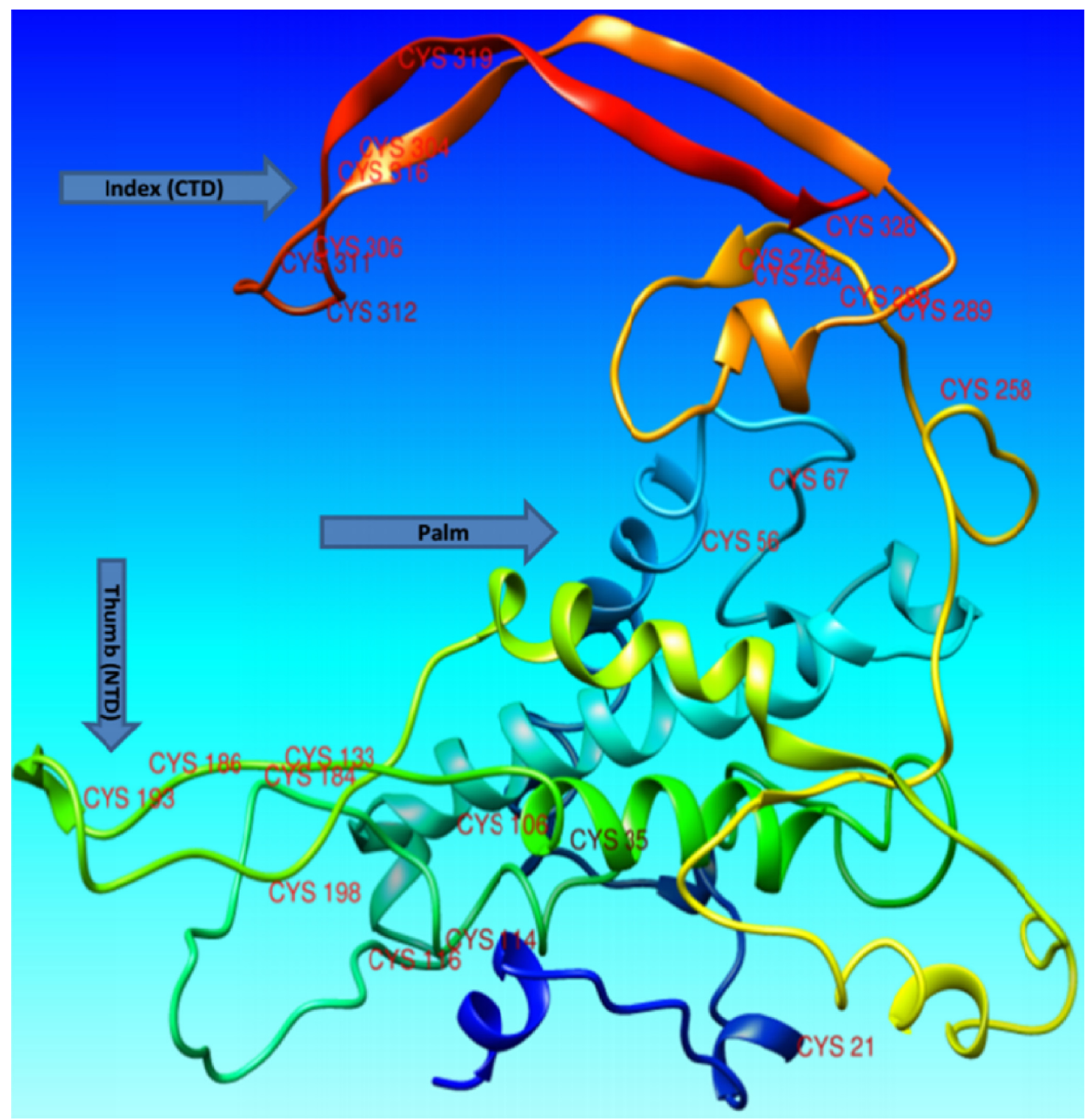

Figure 3 Depiction of a Wnt-4 protein. The Wnt-4 protein contains two distinct thumb and index domains, separated by a palm domain. The cysteine residues are seen to be scattered at the edges of the thumb and index domains and the line joining both domains.

Abbreviations: NTD, N-terminal domain; CTD, C-terminal domain.

antagonist. For better results and increased efficiency, conformers were generated using the OMEGA tool. OMEGA produced 10,531 conformers from 271 unique compounds.

\section{Docking}

The Wnt-4 protein is highly hydrophobic in nature, and the presence of an acyl compound (palmitoleic acid) at Ser190 makes it even more hydrophobic. This palmitoleic acid is also important for correct attachment of Wnt-4 to the frizzled receptor and the efficient activity of the Wnt-4 protein. . $^{59,66}$ Therefore, the docking step was performed in the presence of palmitoleic acid to identify the inhibitor showing maximum interactions with regard to palmitoleic acid and protein residues. Since it is important to make a consensus of compound library for comparative docking experiments, selected docking tools must use the same set of compounds. FRED performs rigid, exhaustive, and non-stochastic molecular docking and filters best molecular pose representing pharmacophoric features. FRED was used to dock conformers library of compounds to Wnt-4. This produced results based on the highest Chemgauss 4 scoring function values. Docking through AD-VINA was applied on 10,531 conformers for 271 compounds. The conformers resulting from OMEGA were also used for docking by GOLD. GOLD uses a genetic algorithm to dock compounds, and ranks compounds on the basis of their Goldscore. ${ }^{67}$ Linux scripts were made to highlight 
and separate the best docked conformer for each unique compound. Scripting resulted in 271 unique compounds that were ranked according to the highest Chemgauss4, binding affinity, and Goldscore scores (Supplementary material 2).

The best compound was selected by comparing the scores for all docking results. For this purpose, the first 15 compounds with the highest Chemgauss4, binding affinity, and Goldscore scores were compared (Table 1). Five compounds having ZINC IDs (ZINC 49543724, ZINC 85876388, ZINC 85876563, ZINC 85878497, and ZINC 85879688 ) were found to be common to all the docking programs (Figure 4). These five compounds were evaluated further on the basis of having more interactions with protein and palmitoleic acid. The interactions were drawn through LigPlot $2^{68}$ and Chimera. ${ }^{69}$ LigPlot 2 interactions were drawn for the selected five compounds and showed that the inhibitor is not only interacting with the protein but also with palmitoleic acid (Supplementary material 3). Only the interactions of 85876365 from AD-VINA, FRED, and GOLD are shown here (Figure 5). Hydrogen bond analysis was also done for the five common protein-inhibitor complexes (Table 2). The major Wnt-4 protein residues involved in these interactions were Ser124, Pro125, Gln126, Gly127, Phe128, Gln129, Ser131, Cys186, His187, Gly188, Val189, Ser190, Gly191, Glu194, Val195, and Thr197. Figure 4 confirms that hydrophobic interactions are dominant, and that hydrogen bond and ionic interactions are also present. The interactions for 85876365 were also drawn through Chimera (Figure 6). Inhibitor was observed to show interactions between palmitoleic acid and Wnt-4 residues, including Lys185, Cys186, His187, Gly188, Val189, Ser190, Gly191, and Ser192. Chimera interactions showed more hydrophobic and less ionic interactions. Palmitoleic acid itself adds extra hydrophobicity to the Wnt-4 protein and maximally supports hydrophobic interactions. A higher degree of hydrophobic interactions might stabilize the protein-inhibitor complex during its passage through the cellular and extracellular environment.

\section{Discussion}

Wnt-4 protein shows many promising structural paradigms that reflect the importance of not only Wnt-4, but also the whole Wnt family. The presence of cysteine residues is a feature of all Wnts. Cysteine residues are very important because of their ability to create cysteine-cysteine disulfide bridges, which provide structural stability. ${ }^{70}$ Cysteine residues are hydrophilic in nature but have hydrophobic characteristics that enable creation of cysteine-cysteine disulfide bonds. ${ }^{71}$ Continuous oxidation-reduction reactions in the cellular and extracellular environment may destroy the protein structure, and disulfide bridges could enable Wnt-4 to withstand a highly oxidative and reductive environment and selectively preserve it even from reactive oxygen species. ${ }^{72}$ Reactive oxygen species readily oxidize free thiol groups on cysteine residues, but if cysteine residues are linked in disulfide bridges, reactive oxygen species may not affect them. ${ }^{73,74}$ Through molecular dynamics simulation studies, it was observed that most of the cysteine residues accumulate near the NTD and CTD, while others fall nearer to the inward line joining the thumb and index domains. This dynamic behavior suggests that cysteine residues are important in the folding mechanism for Wnt-4 protein. The thumb and index domains showed the most conformational variation, while the palm domain show less structural variation. ${ }^{32}$

The thumb domain also has palmitoleic acid bound to it, and is involved in the interaction between Wnt-4 and the frizzled receptor. This finding suggests that the structural importance of Wnt-4 may lie in the dynamic behavior of the thumb-index fold. On the other hand, the activity of Wnt can be indirectly inhibited by inhibiting one of the proteins in the pathway for biosynthesis of Wnts. However, like the Wnt signaling pathway, the Wnt biosynthetic pathway is still poorly understood. The smooth endoplasmic reticulum provides space for palmitoylation of all Wnts. The PORCN enzyme palmitoylates Wnts by adding an acyl adduct. There is no doubt that Wnts are highly conserved proteins, but it has been observed that PORCN tends to add an acyl group at cysteine residues, but that some of the Wnts, including Wnt-4 and XWnt-8, are acylated at the serine residue. ${ }^{75} \mathrm{Wnt}-$ inhibiting mechanisms (WIMs) are being used productively to inhibit Wnt activity. A class of small molecules that were found to disrupt Wnt palmitoylation and secretion along the Wnt/ $\beta$-catenin pathway have been identified as inhibitors of Wnt production (IWP). ${ }^{76,77}$ Wnt activity can be successfully inhibited by inhibiting PORCN through IWPs. ${ }^{78}$ A negative feedback mechanism can also be initiated that might inhibit Wnt activity; for example, excessive addition of Dickkopf protein, ${ }^{79}$ negatively dominant Disheveled or transcription factors/lymphoid enhancer-binding factor, ${ }^{80,81}$ overexpression of intact auxin, or overexpression of full-length glycogen synthase kinase ${ }^{82-85}$ may inhibit Wnt activity in specific circumstances. Another way to inhibit aberrant Wnt activity is to target any of the proteins involved in the signaling and/or biosynthetic pathways for Wnt is to target RNA interference. ${ }^{86}$ All of the proteins involved in the Wnt-inhibiting mechanisms discussed above show general activity, and not a single protein is specific for Wnts. No doubt all these methods can be utilized to inhibit Wnt aberrant expression, but in doing so may have deleterious effects on overall functioning of the cell, and may even cause cancer, and in extreme cases, death of 
Table I The best 15 compounds selected from AD-VINA, FRED, and GOLD

\begin{tabular}{|c|c|c|c|c|c|}
\hline Compound name & AD-VINA-BE & Compound name & $\begin{array}{l}\text { FRED Chemgauss } 4 \\
\text { score }\end{array}$ & Compound name & $\begin{array}{l}\text { GOLD } \\
\text { Goldscore }\end{array}$ \\
\hline ZINC $33834 \mid 33$ & -7.7 & ZINC 85876563 & 8.31026 & ZINC 85876563 & 52.08 \\
\hline ZINC 85879688 & -7.3 & ZINC 85876388 & 8.075899 & ZINC 85878497 & 50.38 \\
\hline ZINC 31034672 & -7.3 & ZINC 85879688 & 7.865263 & ZINC 85879688 & 50.06 \\
\hline ZINC 704543II & -7.2 & ZINC 36378994 & 7.576962 & ZINC 85876388 & 49.54 \\
\hline ZINC 3II67205 & -7.1 & ZINC 0085679I & 7.548942 & ZINC 85875407 & 48.45 \\
\hline ZINC 85876563 & -7 & ZINC 08876343 & 7.468904 & ZINC I3409788 & 48.08 \\
\hline ZINC 0085679I & -7 & ZINC 49543724 & 7.025389 & ZINC I3692706 & 47.3 \\
\hline ZINC 274I569I & -7 & ZINC I2900963 & 6.767407 & ZINC 3I 034672 & 46.87 \\
\hline ZINC 49543724 & -7 & ZINC I 2296500 & 6.7456 & ZINC 49543724 & 46.44 \\
\hline ZINC I 2296700 & -7 & ZINC 31156927 & 6.451456 & ZINC 48057042 & 44.85 \\
\hline ZINC I45I2189 & -7 & ZINC 02101408 & 6.419558 & ZINC 01645309 & 44.8 \\
\hline ZINC 35465238 & -6.9 & ZINC 85875407 & 6.373603 & ZINC 7232603I & 44.74 \\
\hline ZINC 85876388 & -6.8 & ZINC I 2899484 & 6.141479 & ZINC I48I4670 & 44.67 \\
\hline ZINC 85878497 & -6.8 & ZINC 85878497 & 6.12505 & ZINC 03852034 & 44.59 \\
\hline ZINC 36378994 & -6.8 & ZINC 04073 I 74 & 6.106562 & ZINC 03843264 & 44.56 \\
\hline
\end{tabular}

Notes: Values in the table are sorted on the basis of AutoDock binding energy, FRED Chemgauss4, and Goldscore values from top to bottom. It was evident that five compounds were common through all docking programs. These five compounds were then analyzed further to determine out their interactions with palmitoleic acid and Wnt-4 protein. Abbreviation: BE, binding energy; AD-VINA, AutoDock-Vina; FRED, Fast Rigid Exhaustive Docking; GOLD, Genetic Optimization for Ligand Docking.

the cell. Therefore, targeting the complete organism would not be a good choice unless no other option is available.

Liposomally packed antagonists were the first inhibitors designed to directly inhibit aberrant expression of mouse Wnt-3a. It is still not known where these inhibitors bind and what could be the active site of Wnts. In this experiment, we primarily targeted acylated Wnt-4, so that our inhibitors could interact with not only the thumb domain but also with palmitoleic acid. During our experiments, pan-assay interference compounds (PAINS) ${ }^{87}$ were also considered for selection of any ambiguous compounds. Our inhibitors accomplished this task, and readily interacted with Wnt-4 protein, including<smiles>COc1ccc(-c2ccc(=O)n(CC(=O)Nc3cc(OC)c(OC)c(OC)c3)n2)c(F)c1</smiles>

49543724<smiles>COC(=O)C[C@H](c1ccc(OC)c(O)c1)c1oc(CSc2ccccc2)cc(=O)c1O</smiles><smiles>CCc1cc(=O)c(O)c(C)o1</smiles>
S<smiles>CC1=CC=CCC1</smiles>

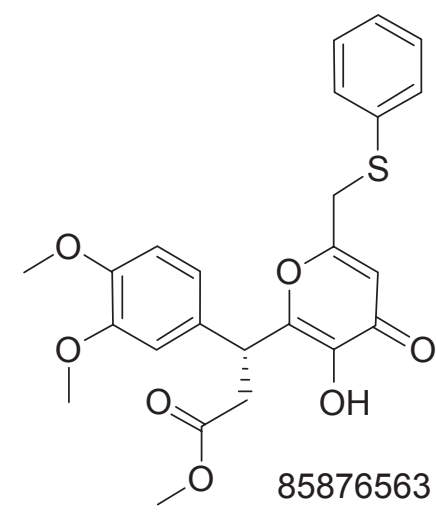

Figure 4 Compounds that resulted from virtual screening and docking analysis. After analyzing the interactions of compounds with Wnt-4 protein, the selected compounds could serve as lead compounds for designing potent drugs. 


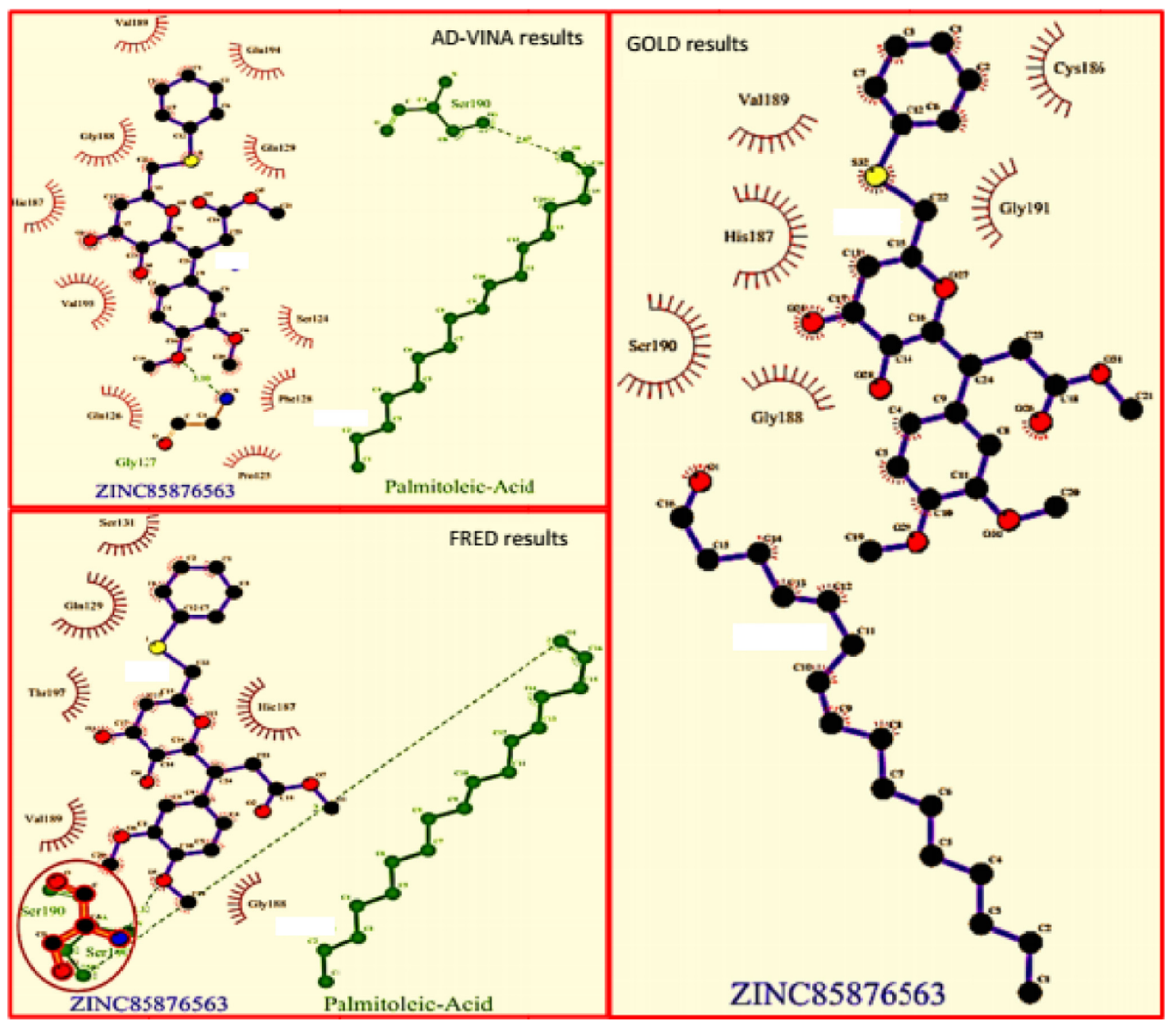

Figure 5 Interaction diagram from GOLD, AutoDock-Vina, and FRED. The inhibitor (ZINC 85876563) interacts with protein residues as well as with palmitoleic acid. The receptor is shown to bind covalently with palmitoleic acid.

Abbreviations: AD-VINA, AutoDock-Vina; FRED, Fast Rigid Exhaustive Docking; GOLD, Genetic Optimization for Ligand Docking.

palmitoleic acid, by dominantly making hydrophobic interactions. These results suggest that our selected inhibitors may serve as lead compounds for further screening, inhibitor searching, and experimentation with Wnt-4 protein. If used in aberrant conditions, the contribution of interactions of our inhibitors with Wnt-4 protein and palmitoleic acid would inhibit Wnt-frizzled signaling cascades. ${ }^{14,88}$

\section{Conclusion}

Wnt proteins show quite different behavior due to high hydrophobicity and number of cysteine - cysteine disulfide bonds, which makes them difficult to study. Computational methods need to be implemented to study the behavior of these proteins, which are diverse and have a distinctive nature. We focused on studying the dynamic behavior of

Table 2 Hydrogen bond analysis

\begin{tabular}{|c|c|c|c|}
\hline ZINC ID & $\begin{array}{l}\text { AD-VINA ( } \mathrm{H} \text { bond) } \\
\text { residues }\end{array}$ & $\begin{array}{l}\text { FRED }(\mathrm{H} \text { bond }) \\
\text { residues }\end{array}$ & $\begin{array}{l}\text { GOLD }(\mathrm{H} \text { bond }) \\
\text { residues }\end{array}$ \\
\hline 49543724 & (0): & (0): & (I): Gly 188 \\
\hline 85876388 & (I): Gly I27 & (0): & (I): Gly 188 \\
\hline 85876563 & (I): Gly I27 & (0): & (I): Ser 190 \\
\hline 85878497 & (2): Ser I90, Hiel 87 & (0): & (2): Ser 190, Gly 188 \\
\hline 85879688 & $\begin{array}{l}\text { (4): Ser 190, Thr I97, } \\
\text { Cys I 86, Hiel } 87\end{array}$ & (0): & (0): \\
\hline
\end{tabular}

Notes: Hydrogen bond analysis was done through UCSF Chimera and LigPlot. Both tools provided the same hydrogen bond results. The residues, Gly 127, Ser 190, Hiel87, Gly 188, Thr 197, and Cys 186 were hot residues for interaction with our inhibitors.

Abbreviations: AD-VINA, AutoDock-Vina; FRED, Fast Rigid Exhaustive Docking; GOLD, Genetic Optimization for Ligand Docking. 

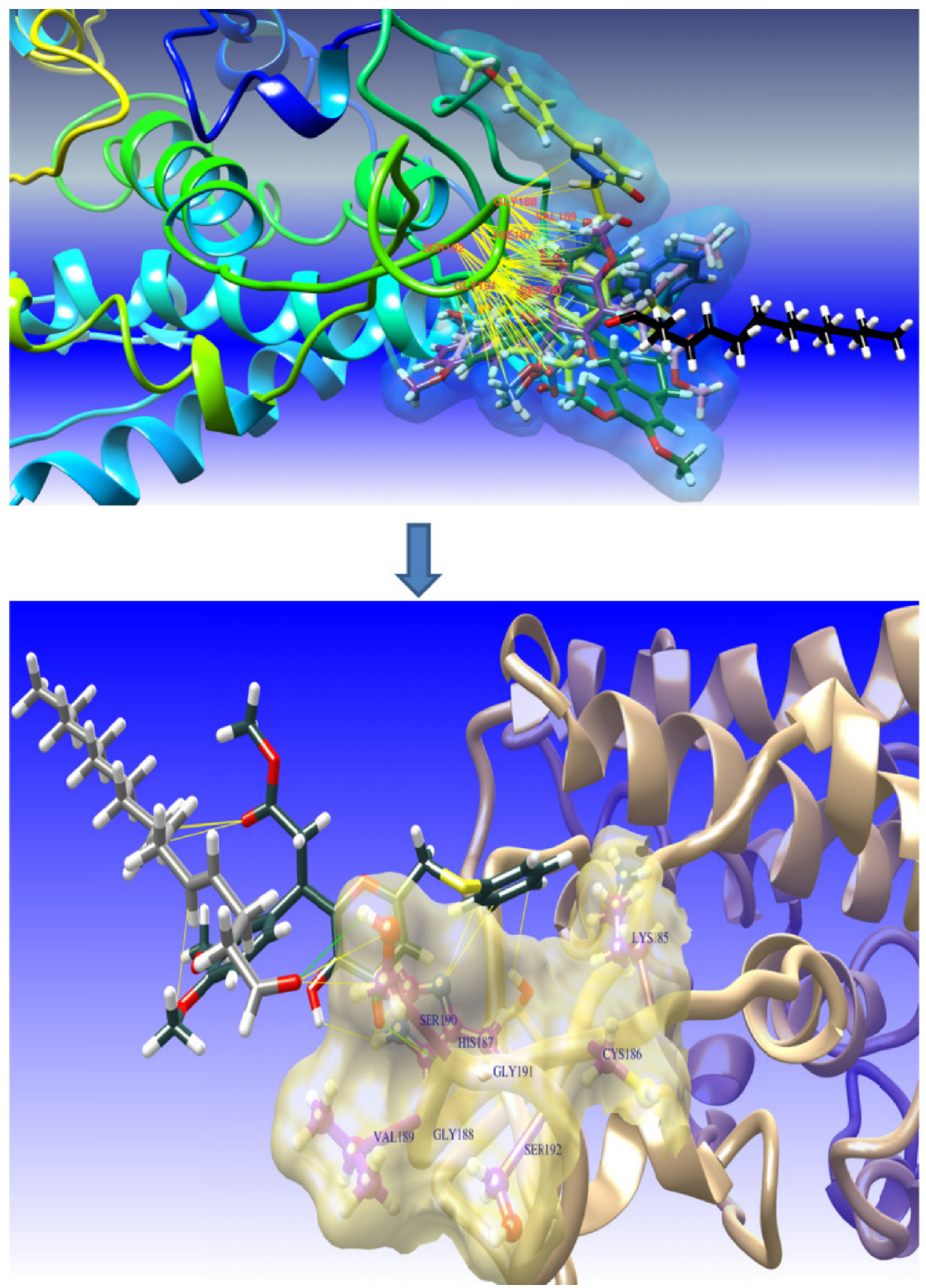

Figure 6 Interactions of the five best compounds with protein and palmitoleic acid. The downward direction of arrow in diagram points to interactions of one selected inhibitor. The interactions of the inhibitor may render Wnt-frizzled activity due to unavailability of palmitoleic acid at the frizzled receptor.

Wnt-4 protein with and without bound inhibitor. Our findings indicate that our inhibitors, selected through VS and comparative docking methodologies, could be the better lead compounds. They can also be useful experimentally as potent drugs. The involvement of an inhibitors with protein residues as well as palmitoleic acid could inhibit Wnt-frizzled pathway. These compounds could also be used to make more active compounds or drugs, particularly in association with Wnt-4 in the canonical and non-canonical signaling cascades. Time-dependent dynamic studies of Wnt-4 revealed many important paradigms of the thumbindex fold and behavior of Wnt-4 protein. The cysteine residues in the thumb-index fold might be important in stabilization of protein, leading to a successful Wnt-4 pathway. Studies incorporating computational and wet laboratory techniques could provide a means for describing the Wnt pathways, designing new inhibitors, and combating irregularities in Wnt. 


\section{Acknowledgment}

The authors are grateful to the Higher Education Commission, Islamabad, Pakistan, for funding this research.

\section{Author contribution}

SSA contributed towards the conception, drafting and revising of the manuscript and MAH helped in data analysis and drafting of the manuscript.

\section{Disclosure}

The authors report no conflicts of interest in this work.

\section{References}

1. Nusse R, Varmust HE. Wnt genes. Cell. 1992;69(7):1073-1087.

2. Parr BA, Shea MJ, Vassileva G, McMahon AP. Mouse Wnt genes exhibit discrete domains of expression in the early embryonic CNS and limb buds. Development. 1993;119(1):247-261.

3. Willert K, Nusse R. Wnt proteins. Cold Spring Harb Perspect Biol. 2012;4(9):a007864.

4. Barker N, Clevers H. Catenins, Wnt signaling and cancer. Bioessays. 2000;22(11):961-965.

5. Kühl M, Sheldahl LC, Park M, Miller JR, Moon RT. The Wnt/Ca2 ${ }^{+}$ pathway. Trends Genet. 2000;16(7):279-283.

6. Lyuksyutova AI, Lu CC, Milanesio N, et al. Anterior-posterior guidance of commissural axons by Wnt-frizzled signaling. Science. 2003;302(5652):1984-1988.

7. Stark K, Vainio S, Vassileva G, McMahon AP. Epithelial transformation of metanephric mesenchyme in the developing kidney regulated by Wnt-4. Nature. 1994;372(6507):679-683.

8. Lyons JP, Mueller UW, Ji H, et al. Wnt-4 activates the canonical betacatenin-mediated Wnt pathway and binds Frizzled-6 CRD: functional implications of Wnt/beta-catenin activity in kidney epithelial cells. Exp Cell Res. 2004:298(2):369-387.

9. Kispert A, Vainio S, McMahon A. Wnt-4 is a mesenchymal signal for epithelial transformation of metanephric mesenchyme in the developing kidney. Development. 1998;125(21):4225-4234.

10. Gavin BJ, McMahon JA, McMahon AP. Expression of multiple novel Wnt-1/int-1-related genes during fetal and adult mouse development. Genes Dev. 1990;4(12B):2319-2332.

11. Maurus D, Heligon C, Burger-Schwarzler A, Brandli AW, Kuhl M. Noncanonical Wnt-4 signaling and EAF2 are required for eye development in Xenopus laevis. EMBO J. 2005;24(6):1181-1191.

12. Kim Y, Kobayashi A, Sekido R, et al. Fgf9 and Wnt4 act as antagonistic signals to regulate mammalian sex determination. PLoS Biol. 2006;4(6):1000-1009.

13. Biason-Lauber A, Konrad D, Navratil F, Schoenle EJ. A WNT4 mutation associated with Mullerian-duct regression and virilization in a 46,XX woman. $N$ Engl J Med. 2004;351(8):792-798.

14. Herr P, Hausmann G, Basler K. WNT secretion and signalling in human disease. Trends Mol Med. 2012;18(8):483-493.

15. Hausmann G, Basler K. Wnt lipid modifications: not as saturated as we thought. Dev Cell. 2006;11(6):751-752.

16. McMahon AP, Moon RT. Int-1 - a proto-oncogene involved in cell signaling. Development. 1989;107:161-167.

17. Janda CY, Waghray D, Levin AM, Thomas C, Garcia KC. Structural basis of Wnt recognition by frizzled. Science. 2012;337(6090):59-64.

18. Chu M, Ahn V, Choi H, Daniels D. Structural studies of Wnts and identification of an LRP6 binding site. Structure. 2013;21(7):1235-1242.

19. Putnam NH, Srivastava M, Hellsten U, et al. Sea anemone genome reveals ancestral eumetazoan gene repertoire and genomic organization. Science. 2007;317(5834):86-94.

20. Niehrs C, Acebron SP. Mitotic and mitogenic Wnt signalling. EMBOJ. 2012;31(12):2705-2713.
21. Schwarz-Romond T. Three decades of Wnt signalling. EMBO J. 2012;31(12):2664.

22. Watanabe K, Dai X. Winning WNT: race to Wnt signaling inhibitors. Proc Natl Acad Sci U S A. 2011;108(15):5929-5930.

23. Croce JC, McClay DR. Evolution of the Wnt pathways. In: Wnt Signaling. Berlin, Germany: Springer; 2009.

24. Morrell NT, Leucht P, Zhao LD, et al. Liposomal packaging generates Wnt protein with in vivo biological activity. PLoS One. 2008;3(8): e2930.

25. Shea J-E, Brooks CL III. From folding theories to folding proteins: a review and assessment of simulation studies of protein folding and unfolding. Annu Rev Phys Chem. 2001;52(1):499-535.

26. Voelz VA, Bowman GR, Beauchamp K, Pande VS. Molecular simulation of ab initio protein folding for a millisecond folder NTL9 (1-39). J Am Chem Soc. 2010;132(5):1526-1528.

27. Kitchen DB, Decornez H, Furr JR, Bajorath J. Docking and scoring in virtual screening for drug discovery: methods and applications. Nat Rev Drug Discov. 2004;3(11):935-949.

28. McGann MR, Almond HR, Nicholls A, Grant JA, Brown FK. Gaussian docking functions. Biopolymers. 2003;68(1):76-90.

29. Kroemer RT, Vulpetti A, McDonald JJ, et al. Assessment of docking poses: interactions-based accuracy classification (IBAC) versus crystal structure deviations. J Chem Inf Comput Sci. 2004;44(3):871-881.

30. Leach A, Shoichet B, Peishoff C. Docking and scoring. J Med Chem. 2006;49(20):5851-5856.

31. Warren GL, Andrews CW, Capelli A-M, et al. A critical assessment of docking programs and scoring functions. $J$ Med Chem. 2006;49(20):5912-5931.

32. Azam SS, Hammad MA. Role of thumb index fold in Wnt-4 protein and its dynamics through a molecular dynamics simulation study. J Mol Liq. 2014;198:313-321.

33. Ogbonna N. Molecular Dynamics Simulation. Muizenberg: African Institute for Mathematical Sciences; 2004.

34. Barrett JH. Breakdown of the statistical-equilibrium hypothesis in channeling. Phys Rev Lett. 1973;31(26):1542-1545.

35. Thirumalai D, Mountain RD. Ergodic convergence properties of supercooled liquids and glasses. Phys Rev A. 1990;42(8):4574-4587.

36. Irwin JJ, Shoichet BK. ZINC - a free database of commercially available compounds for virtual screening. J Chem Inf Model. 2005; 45(1):177-182

37. Fersht AR, Daggett V. Protein folding and unfolding at atomic resolution. Cell. 2002;108(4):573-582.

38. Fersht AR. Optimization of rates of protein folding: the nucleationcondensation mechanism and its implications. Proc Natl Acad Sci U S A. 1995;92(24):10869-10873.

39. Dobson CM. Protein folding and misfolding. Nature. 2003;426(6968): 884-890.

40. Eswar N, John B, Mirkovic N, et al. Tools for comparative protein structure modeling and analysis. Nucleic Acids Res. 2003;31(13):3375-3380.

41. Snow CD, Sorin EJ, Rhee YM, Pande VS. How well can simulation predict protein folding kinetics and thermodynamics? Annu Rev Biophys Biomol Struct. 2005;34(1):43-69.

42. OEChem TK. Version 1.7. 4.3; OpenEye Scientific Software Inc. St Fe, NM. 2010.

43. Choi J, He N, Kim N, Yoon S. Enrichment of virtual hits by progressive shape-matching and docking. J Mol Graph Model. 2012;32:82-88.

44. Lyne PD. Structure-based virtual screening: an overview. Drug Discov Today. 2002;7(20):1047-1055.

45. Reddy AS, Pati SP, Kumar PP, Pradeep HN, Sastry GN. Virtual screening in drug discovery - a computational perspective. Curr Protein Pept Sci. 2007;8(4):329-351.

46. Muchmore SW, Souers AJ, Akritopoulou-Zanze I. The use of threedimensional shape and electrostatic similarity searching in the identification of a melanin-concentrating hormone receptor 1 antagonist. Chem Biol Drug Des. 2006;67(2):174-176.

47. Tresadern G, Bemporad D, Howe T. A comparison of ligand based virtual screening methods and application to corticotropin releasing factor 1 receptor. J Mol Graph Model. 2009;27(8):860-870. 
48. Venkatraman V, Pérez-Nueno VI, Mavridis L, Ritchie DW. Comprehensive comparison of ligand-based virtual screening tools against the DUD data set reveals limitations of current 3D methods. J Chem Inf Model. 2010;50(12):2079-2093.

49. Sala E, Guasch L, Iwaszkiewicz J, et al. Identification of human IKK-2 inhibitors of natural origin (part I): modeling of the IKK-2 kinase domain, virtual screening and activity assays. PLoS One. 2011;6(2):e16903.

50. Hawkins PC, Skillman AG, Warren GL, Ellingson BA, Stahl MT. Conformer generation with OMEGA: algorithm and validation using high quality structures from the Protein Databank and Cambridge Structural Database. J Chem Inf Model. 2010;50(4):572-584.

51. McGann M. FRED pose prediction and virtual screening accuracy. J Chem Inf Model. 2011;51(3):578-596.

52. McGaughey GB, Sheridan RP, Bayly CI, et al. Comparison of topological, shape, and docking methods in virtual screening. J Chem Inf Model. 2007;47(4):1504-1519.

53. Trott O, Olson AJ. AutoDock Vina: improving the speed and accuracy of docking with a new scoring function, efficient optimization, and multithreading. J Comput Chem. 2010;31(2):455-461.

54. Bikadi Z, Hazai E. Application of the PM6 semi-empirical method to modeling proteins enhances docking accuracy of AutoDock. J Cheminform. 2009;1:15.

55. Morris GM, Huey R, Olson AJ. Using AutoDock for ligand-receptor docking. Curr Protoc Bioinformatics. 2008; Chapter 8:Unit 8.14.

56. Verdonk ML, Cole JC, Hartshorn MJ, Murray CW, Taylor RD. Improved protein-ligand docking using GOLD. Proteins. 2003;52(4):609-623.

57. Kontoyianni M, McClellan LM, Sokol GS. Evaluation of docking performance: comparative data on docking algorithms. J Med Chem. 2004;47(3):558-565

58. Fidelis K, Adzhubej A, Kryshtafovych A, Daniluk P. Protein Model Database. Livermore, CA, USA: Lawrence Livermore National Laboratory; 2005.

59. Takada R, Satomi Y, Kurata T, et al. Monounsaturated fatty acid modification of Wnt protein: its role in Wnt secretion. Dev Cell. 2006;11(6):791-801.

60. Banziger C, Soldini D, Schutt C, Zipperlen P, Hausmann G, Basler K. Wntless, a conserved membrane protein dedicated to the secretion of Wnt proteins from signaling cells. Cell. 2006;125(3):509-522.

61. Hert J, Irwin JJ, Laggner C, Keiser MJ, Shoichet BK. Quantifying biogenic bias in screening libraries. Nat Chem Biol. 2009;5(7):479-483.

62. Newman DJ, Cragg GM. Natural products as sources of new drugs over the last 25 years. J Nat Prod. 2007;70(3):461-477.

63. Mirza MU, Mirza AH, Ghori N, Ferdous S. Glycyrrhetinic acid and E. resveratroloside act as potential plant derived compounds against dopamine receptor D3 for Parkinson's disease: a pharmacoinformatics study. Drug Des Devel Ther. 2014;9:187-198.

64. Jonckers TH, Lin TI, Buyck C, et al. 2'-Deoxy-2'-spirocyclopropylcytidine revisited: a new and selective inhibitor of the hepatitis C virus NS5B polymerase. J Med Chem. 2010;53(22):8150-8160.

65. Boström J, Grant JA, Fjellström O, Thelin A, Gustafsson D. Potent fibrinolysis inhibitor discovered by shape and electrostatic complementarity to the drug tranexamic acid. J Med Chem. 2013;56(8):3273-3280.

66. Nadolski MJ, Linder ME. Protein lipidation. FEBS J. 2007;274(20): 5202-5210.

67. Marcou G, Kellenberger E. Docking with GOLD. Available from: http:// infochimie.u-strasbg.fr/master/cours25112009.pdf. Accessed March 12, 2015 .

Drug Design, Development and Therapy

\section{Publish your work in this journal}

Drug Design, Development and Therapy is an international, peerreviewed open-access journal that spans the spectrum of drug design and development through to clinical applications. Clinical outcomes, patient safety, and programs for the development and effective, safe, and sustained use of medicines are a feature of the journal, which
68. Wallace AC, Laskowski RA, Thornton JM. LigPlot: a program to generate schematic diagrams of protein-ligand interactions. Protein Eng. 1995;8(2):127-134.

69. Pettersen EF, Goddard TD, Huang CC, et al. UCSF Chimera - a visualization system for exploratory research and analysis. J Comput Chem. 2004;25(13):1605-1612.

70. Bulaj G. Formation of disulfide bonds in proteins and peptides. Biotechnol Adv. 2005;23(1):87-92.

71. Raina S, Missiakas D. Making and breaking disulfide bonds. Annu Rev Microbiol. 1997;51(1):179-202.

72. Miki H, Funato Y. Regulation of intracellular signalling through cysteine oxidation by reactive oxygen species. J Biochem. 2012;151(3):255-261.

73. Boronat $\mathrm{S}$, Domènech $\mathrm{A}$, Paulo $\mathrm{E}$, et al. Thiol-based $\mathrm{H}_{2} \mathrm{O}_{2}$ signalling in microbial systems. Redox Biol. 2014;2:395-399.

74. Zhang C-M, Christian T, Newberry KJ, Perona JJ, Hou Y-M. Zincmediated amino acid discrimination in cysteinyl-tRNA synthetase. J Mol Biol. 2003;327(5):911-917.

75. Proffitt KD, Madan B, Ke Z, et al. Pharmacological inhibition of the Wnt acyltransferase PORCN prevents growth of WNT-driven mammary cancer. Cancer Res. 2013;73(2):502-507.

76. Chen W, Chen M, Barak L. Development of small molecules targeting the Wnt pathway for the treatment of colon cancer: a high-throughput screening approach. Am J Physiol. 2010;27710(25):293-300.

77. Chen B, Dodge M, Tang W, Lu J. Small molecule-mediated disruption of Wnt-dependent signaling in tissue regeneration and cancer. Nat Chem. 2009;5(2):100-107.

78. Dodge M, Lum L. Drugging the cancer stem cell compartment: lessons learned from the hedgehog and Wnt signal transduction pathways. Annu Rev Pharmacol Toxicol. 2011;51:289-310.

79. Glinka A, Wu W, Delius H, Monaghan AP, Blumenstock C, Niehrs C. Dickkopf-1 is a member of a new family of secreted proteins and functions in head induction. Nature. 1998;391(6665):357-362.

80. Molenaar M, Vandewetering M, Oosterwegel M, et al. XTcf-3 transcription factor mediates $\beta$-catenin-induced axis formation in xenopus embryos. Cell. 1996;86(3):391-399.

81. Wallingford JB, Rowning BA, Vogeli KM, Rothbacher U, Fraser SE, Harland RM. Dishevelled controls cell polarity during Xenopus gastrulation. Nature. 2000;405(6782):81-85.

82. Willert K, Logan CY, Arora A, Fish M, Nusse R. A Drosophila axin homolog, daxin, inhibits Wnt signaling. Development. 1999;126(18): 4165-4173.

83. He X, Saint-Jeannet J-P, Woodgett JR, Varmus HE, Dawid IB. Glycogen synthase kinase-3 and dorsoventral patterning in Xenopus embryos. Nature. 1995;374(6523):617-622.

84. Zeng L, Fagotto F, Zhang T, et al. The mouse fused locus encodes axin, an inhibitor of the Wnt signaling pathway that regulates embryonic axis formation. Cell. 1997;90(1):181-192.

85. Itoh K, Krupnik VE, Sokol SY. Axis determination in Xenopus involves biochemical interactions of axin, glycogen synthase kinase 3 and ß-catenin. Curr Biol. 1998;8(10):591-594.

86. Gurney A, Axelrod F, Bond CJ, et al. Wnt pathway inhibition via the targeting of Frizzled receptors results in decreased growth and tumorigenicity of human tumors. Proc Natl Acad Sci U S A. 2012;109(29):11717-11722.

87. Baell J, Walters MA. Chemistry: chemical con artists foil drug discovery. Nature. 2014;513(7519):481-483.

88. Altchek A, Deligdisch L. The unappreciated Wnt-4 gene. J Pediatr Adolesc Gynecol. 2010;23(3):187-191.

\section{Dovepress}

has also been accepted for indexing on PubMed Central. The manuscript management system is completely online and includes a very quick and fair peer-review system, which is all easy to use. Visit http://www.dovepress.com/testimonials.php to read real quotes from published authors. 